\title{
DISCOUNTED AND POSITIVE STOCHASTIC GAMES
}

\author{
BY T. PARTHASARATHY
}

Communicated by M. H. Protter, May 11, 1970

1. Introduction. The main purpose of this note is to announce a few results on stochastic games. A stochastic game is determined by five objects: $S, A, B, q$ and $r . S, A$ and $B$ are nonempty Borel Subsets of Polish spaces and $r$ is a bounded measurable function on $S \times A \times B$. We interpret $S$ as the state space of some system and $A, B$ as the set of actions available to players I and II respectively at each state. When the system is in state $s$ and players I and II choose action $a$ and $b$ respectively, the system moves to a new state according to the distribution $q(\cdot \mid s, a, b)$ and I receives from II, $r(s, a, b)$ units of money. Then the whole process is repeated from the new state $s^{\prime}$. The problem, then, is to maximize player I's expected income as the game proceeds over the infinite future and to minimize player II's expected loss.

A strategy $\pi$ for player $I$ is a sequence $\pi_{1}, \pi_{2}, \cdots$, where $\pi_{n}$ specifies the action to be chosen by player I on the $n$th day by associating (Borel measurably) with each history

$$
h=\left(s_{1}, a_{1}, b_{1}, \cdots, s_{n-1}, a_{n-1}, b_{n-1}, s_{n}\right)
$$

of the system a probability distribution $\pi_{n}(\cdot \mid h)$ on the Borel sets of $A$. Call $\pi$ a stationary strategy if there is a Borel map $f$ from $S$ to $P_{A}$, where $P_{A}$ is the set of all probability measures on the Borel sets of $A$, such that $\pi_{n}=f$ for each $n \geqq 1$ and in this case, $\pi$ is denoted by $f^{(\infty)}$. Strategies and stationary strategies are defined similarly for II.

Let $\beta$ be any fixed nonnegative number satisfying $0 \leqq \beta<1$. A pair $(\pi, \Gamma)$ of strategies for I and II associates with each initial state $s$, a $n$th day expected income $r_{n}(\pi, \Gamma)(s)$ for I and a total expected discounted income

$$
I_{\beta}(\pi, \Gamma)(s)=\sum_{n=1}^{\infty} \beta^{n-1} r_{n}(\pi, \Gamma)(s)
$$

Such stochastic games are called discounted stochastic games. Positive stochastic games are those where $r(s, a, b) \geqq 0 \forall s, a, b$ and $\beta=1$.

AMS 1969 subject classifications. Primary 9070; Secondary 9070, 9072.

Key words and phrases. Stochastic games, discounted, positive, optimal stationary strategies. 
Call $\pi^{*}$ optimal for I if

$$
I_{\beta}\left(\pi^{*}, \Gamma\right)(s) \geqq \inf _{\Gamma} \sup _{\boldsymbol{\tau}} I_{\beta}(\pi, \Gamma)(s)
$$

for all $\Gamma$ and $s$. Call $\Gamma^{*}$ optimal for II if

$$
I_{\beta}\left(\pi, \Gamma^{*}\right)(s) \leqq \sup _{\boldsymbol{\tau}} \inf _{\boldsymbol{\Gamma}} I_{\beta}(\pi, \Gamma)(s)
$$

for all $\pi$ and $s$. We shall say that the stochastic game has a value if

$$
\sup _{\boldsymbol{\tau}} \inf _{\mathbf{\Gamma}} I_{\boldsymbol{\beta}}(\pi, \Gamma)(s)=\inf _{\mathbf{\Gamma}} \sup _{\boldsymbol{\pi}} I_{\boldsymbol{\beta}}(\pi, \Gamma)(s)
$$

for all $s$.

The case where the stochastic game has a value, sup inf $I_{\beta}(\pi, \Gamma)(s)$, as a function of $S$ is called the value function.

2. Main results. Throughout this paper the following assumptions unless otherwise stated will remain operative.

(i) $S$ will be a complete separable metric space; $A$ and $B$ finite sets.

(ii) The following multifunctions are measurable.

$$
\begin{gathered}
r_{w}: S \rightarrow P_{A} \times P_{B}, \\
r_{w}(s)=\left\{\left(\mu^{\prime}, \lambda^{\prime}\right): \max _{\mu}\left[r\left(s, \mu, \lambda^{\prime}\right)+\beta \int w(\cdot) d q\left(\cdot \mid s, \mu, \lambda^{\prime}\right)\right]=\right. \\
\left.\min _{\lambda}\left[r\left(s, \mu^{\prime}, \lambda\right)+\beta \int w(\cdot) d q\left(\cdot \mid s, \mu^{\prime}, \lambda\right)\right]\right\}
\end{gathered}
$$

where $w \in M(s)=$ space of bounded Borel measurable functions on $S$ and $\beta \in[0,1]$.

Now we are in a position to state our theorems.

THEOREM 1. Let $S$ be a complete separable metric space and $A, B$ are finite sets. Further suppose the mutifunctions $\left\{r_{w}\right\}$ are measurable. Then the discounted stochastic game has a value and the value function is Borel measurable. Furthermore players I and II have optimal stationary strategies.

THEOREM 2. Let $S$ be a complete separable metric space and $A$ and $B$ are compact metric. Suppose that, whenever $\left(a_{n}, b_{n}\right) \rightarrow\left(a_{0}, b_{0}\right)$ in $A \times B$, $r\left(s, a_{n}, b_{n}\right) \rightarrow r\left(s, a_{0}, b_{0}\right)$ and $\int w(\cdot) d q\left(\cdot \mid s, a_{n}, b_{n}\right) \rightarrow \int w(\cdot) d q\left(\cdot \mid s, a_{0}, b_{0}\right)$ for every s and w. Further assume that the multifunctions $\left\{r_{w}\right\}$ are measurable. Then the discounted stochastic game has a value and the value function is measurable. Also the two players have optimal stationary strategies. 
THEOREM 3. Let $S$ be a complete separable metric space and $A, B$ are finite sets. Let $r(s, a, b) \geqq 0$ for all $s, a$ and $b$. Suppose $I(\pi, \Gamma)(s) \leqq R$ for all $\pi, \Gamma$ and $s$ where $R$ is a positive real number independent of $\pi, \Gamma$ and $s$. If $\left\{r_{w}\right\}$ are measurable then the positive stochastic game has a value and the value function is measurable.

REMARK 1. Proof of Theorem 1 (as well as the other two theorems) depends on a selection theorem that was proved recently by C. J. Himmelberg and F. S. Van Vleck (see [2, Theorem 4, p. 396]).

REMARK 2. We will not attempt to prove these theorems for they follow along similar lines to that of Theorem 4.1 in [3], where we assumed $S, A, B$ are compact metric and $r(s, a, b), q(\cdot \mid s, a, b)$ are continuous in $S \times A \times B$.

REMARK 3. In Theorem 3 we can also prove that the minimizing player has an optimal stationary strategy using a result of Blackwell on positive dynamic programming (see [1, Theorem 2, p. 416]).We have not been able to determine, whether or not, under our conditions, player I has an optimal stationary strategy.

Acknowledgment. The author is grateful to Professors: R. J. Aumann and A. Maitra for some useful suggestions.

\section{REFERENCES}

1. D. Blackwell, Positive dynamic programming, Proc. Fifth Berkeley Sympos. Math. Statist. and Probability (Berkeley, Calif., 1965) vol. 1: Statistics, Univ. of California Press, Berkeley, Calif., 1967, pp. 415-418. MR 36 \#1193.

2. C. J. Himmelberg and F. S. Van Vleck, Some selection theorems for measurable functions, Canad. J. Math. 22 (1969), 394-397. MR 38 \#4637.

3. A. Maitra and T. Parthasarathy, On stochastic games, J. Optimization Theory Appl. 5 (1970), 289-300.

Case Western Reserve University, Cleveland, Ohio 44106

Indian Statistical Institute, Calcutta, India 